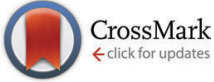

Cite this: DOI: $10.1039 / \mathrm{c5}$ cp05240h

\title{
Probing the effects of 2D confinement on hydrogen dynamics in water and ice adsorbed in graphene oxide sponges $\dagger$
}

\author{
Giovanni Romanelli, + $^{\text {a }}$ Roberto Senesi, ${ }^{\text {ab }}$ Xuan Zhang, ${ }^{c}$ Kian Ping Loh $^{c}$ and \\ Carla Andreani ${ }^{\mathrm{a}}$
}

\begin{abstract}
We studied the single particle dynamics of water and ice adsorbed in graphene oxide (GO) sponges at $T=293 \mathrm{~K}$ and $T=20 \mathrm{~K}$. We used Deep Inelastic Neutron Scattering (DINS) at the ISIS neutron and muon spallation source to derive the hydrogen mean kinetic energy, $\left\langle E_{\mathrm{K}}\right\rangle$, and momentum distribution, $n(p)$. The goal of this work was to study the hydrogen dynamics under 2D confinement and the potential energy surface, fingerprinting the hydrogen interaction with the layered structure of the GO sponge. The observed scattering is interpreted within the framework of the impulse approximation. Samples of both water and ice adsorbed in GO show $n(p)$ functions with almost harmonic and anisotropic line shapes and $\left\langle E_{K}\right\rangle$ values in excess of the values found at the corresponding temperatures in the bulk. The hydrogen dynamics are discussed in the context of the interaction between the interfacial water and ice and the confining hydrophilic surface of the GO sponge.
\end{abstract}

Received 2nd September 2015 Accepted 29th October 2015

DOI: $10.1039 / c 5 c p 05240 h$

www.rsc.org/pccp mainly characterized by an inhomogeneous local density distribution, whereas the dynamics shows an increasing anisotropic behaviour in translational and rotational molecular motions.

Graphene oxide (GO) is a nonstoichiometric compound ${ }^{23}$ of great interest for proposed applications for battery electrodes ${ }^{24,25}$ and membrane models. ${ }^{26-28}$ Previous work on GO has shown that graphene monolayers can be used to sandwich a layer of water film, which remains trapped under vacuum and at high temperatures due to the impermeability of graphene, thus allowing an opportunity to study the molecular structure and dynamics of water under superheated or supercritical conditions. ${ }^{29}$ This study showed how a sprinkle of graphene oxide nanoflakes on graphene is effective at condensing water nanodroplets and seeding ice epitaxy at ambient conditions, with observation of the complex interplay of ionic and non-ionic interactions. By controlling the relative humidity and nanoflake density, the formation of a complete ice wetting layer is slowed down to a time scale of 20 hours. This presented an unprecedented opportunity to visualize ice nucleation and growth in real time and at the molecular level, which can be observed using non-contact atomic force microscopy. ${ }^{30}$ Elucidation of the local environment of hydrogen in water and ice is particularly relevant due to the interplay between the crucial role of protons in hydrogen bonding and the perturbation induced by the two dimensional (2D) confining substrates. In particular the understanding of hydrogen bonding dynamics in GO is important due to the technological relevance of the material. As an example, a $\mathrm{pH}$-sensitive graphene oxide composite hydrogel has been made and utilized for selective drug release at physiological $\mathrm{pH}^{31}$ 
In this paper we present the results of a Deep Inelastic Neutron Scattering (DINS) study of the hydrogen dynamics of water and ice 2D-adsorbed in GO layered sponges at $T=293 \mathrm{~K}$ and $T=20 \mathrm{~K}$, using neutrons with high momentum, $\hbar q$, and energy transfers, $\hbar \omega$. Information about the water hydrogen dynamics is crucial for the understanding of the structure of the carbon monolayers as well as the intercalation process. A FTIR characterization of the dry GO sponges shows that its hydrogen amount is indeed very small (for details see the ESI $\dagger$ ). This guarantees that the hydrogen dynamics probed in the present work arises from the adsorbed water only.

DINS probes the quantum behaviour of atomic nuclei directly through the single-particle momentum distribution, $n(p)$, and the mean kinetic energy, $\left\langle E_{\mathrm{K}}\right\rangle$. These quantities fingerprint the nuclear quantum effects determined by the properties of the ground state. $^{32,33}$ In the case of ice or water, chemical interactions occurring in the bulk typically represent small changes in the energy of constituents, compared to the energy sequestered in the zero-point motion of the protons, primarily in that of the stretching mode. Nuclear quantum effects significantly impact both structure and dynamics and zero-point energy changes as the structure of the hydrogen bond network changes. Thus, because of the non-commuting character of position and momentum operators in quantum mechanics, the $n(p)$ is a sensitive probe to the hydrogen local environment as well as a direct measurement of its dynamics. ${ }^{32,34-37,39}$ It is important to emphasize the difficulty in obtaining similar information regarding the proton dynamics by other techniques, such as Inelastic Neutron Scattering (INS) or Infrared (IR). In these cases they fingerprint different properties related to the vibrational transitions from the ground state to the first excited states. ${ }^{38}$ Thus the $n(p)$ measured via DINS is the only quantity which probes the underlying potential energy surface that the hydrogen in water sees as the local environment changes and water adapts its hydrogen-bonding network in response to $2 \mathrm{D}$ confinement in GO sheets.

The DINS technique, also known as Neutron Compton Scattering (NCS), ${ }^{32,40}$ probes time-scale regimes in the order of attoseconds, i.e., $10^{-15}-10^{-17} \mathrm{~s}$, where scattering is entirely incoherent, i.e., a time window much shorter than the time constants characteristic of the typical collective excitations, typically well above $10^{-15} \mathrm{~s}^{41}$

The basic principles of data interpretation of the DINS technique are based on the validity of the Impulse Approximation (IA) ${ }^{42}$ which is exact in the limit of infinite momentum transfer. ${ }^{43,44}$ Within the IA, the inelastic neutron scattering cross-section directly probes the $n(p)$ of all nuclei in the target system and elucidates its connection with the underlying potential energy surface. ${ }^{32}$ The $n(p)$ line-shape fingerprints the details of the 2D confining potential energy surface that the protons experience in contact with the GO surface, directly reflecting the structure of their local environment. DINS results complement information which is garnered from diffraction techniques that measure the spatial correlations among the nuclear positions. From the results of the analysis of the DINS data, we are able to capture the proton dynamics of water and ice adsorbed in GO and obtain new information on the potential energy surface experienced by the proton. Section 2 illustrates DINS experiments and data analysis. In Section 3, results are presented and discussed. Conclusions are reported in Section 4 .

\section{Experiment}

DINS measurements were performed at the ISIS neutron and muon spallation source (Rutherford Appleton Laboratory, Chilton, Didcot, UK) on the inverse geometry spectrometer VESUVIO ${ }^{40,45,46}$ that uses neutrons with incident energies in the range $1-10^{4} \mathrm{eV}$. The experiment was performed on hydrated samples of water and ice adsorbed in dry GO sponges contained in an $\mathrm{Al}$ container at two different temperatures: at $T=293 \mathrm{~K}$ and at $T=20 \mathrm{~K}$, at ambient pressure. The sample with water fully absorbed in the GO sponge had a total weight of $1.340 \mathrm{~g}$. The weights of the hydrated sample, i.e., the adsorbed water and the dry GO were $1.172 \mathrm{~g}$ and $0.168 \mathrm{~g}$, respectively, corresponding to approximately $87 \mathrm{wt} \%$ of adsorbed water. Sample preparation materials, a detailed description of the operation of the VESUVIO instrument and data analysis are described in the ESI. $\dagger$

The neutron scattering function in the IA regime, $S_{\mathrm{IA}}(q, \omega)$ is expressed in terms of the West scaling variable ${ }^{42}$ by:

$$
\frac{\hbar q}{m} S_{\mathrm{IA}}(\mathbf{q}, \omega)=J_{\mathrm{IA}}(y, \hat{\mathbf{q}})=\int n(\mathbf{p}) \delta(y-\mathbf{p} \cdot \hat{\mathbf{q}}) \mathrm{d} \mathbf{p}
$$

where $m$ is the mass of the particle being struck by the neutron,

$$
y_{m}=\frac{m}{\hbar q}\left(\omega-\frac{\hbar q^{2}}{2 m}\right)
$$

$n(\mathbf{p})$ is the projection of the particle momentum distribution along the $\hat{\mathbf{q}}$ direction, and $J_{\mathrm{IA}}(y, \hat{\mathbf{q}})$ is the neutron Compton Profile (NCP). $\S^{32}$ When the sample is isotropic, the particle momentum distribution only depends on the modulus of $\mathbf{p}$, and the $\hat{\mathbf{q}}$ direction is immaterial, so the NCP is simply $J_{\mathrm{IA}}(y)=2 \pi \int_{|y|}^{\infty} p n(p) \mathrm{d} p$. This ideal peak profile, measured in a DINS experiment, is broadened by finite- $q$ correction terms $\Delta J(y, q)$, and by convolution with the instrumental resolution function $R(y, q)$, so the experimental NCP, $F(y, q)$, yields:

$$
F(y, q)=\left[J_{\mathrm{IA}}(y)+\Delta J(y, q)\right] \star R(y, q),
$$

with $\Delta J(y, q) \propto \frac{\partial^{3}}{\partial y^{3}} J_{\mathrm{IA}}(y)$ (as described in the $\operatorname{ESI} \dagger$ ) and $R(y, q)$ determined using Monte Carlo routines available on VESUVIO.

\section{Data analysis and discussion}

The primary goal of this experiment was to derive the $J(y)$ lineshape from the $F(y, q)$ spectra and subsequently calculate the $n(p)$ and $\left\langle E_{\mathbf{K}}\right\rangle$ of the adsorbed water's hydrogens. GO substrates are particularly favourable in these respects, in that the hydrogen amount in the dry GO sponges is very small, as confirmed by the results of the FTIR characterization. DINS measurements of the dry GO sponges confirm this finding: the hydrogen signal from the dry GO is negligible as compared to the hydrated

$\S$ For consistency with previous literature and ease of notation we write the momentum as a wave vector. 
samples (see Fig. S8 in ESI† for details). This guarantees that DINS measures the dynamics of the protons in the $\mathrm{H}_{2} \mathrm{O}$ adsorbed in the GO sponge only. A global fit of the individual $F(y, q)$ spectra, recorded for the angles in forward scattering, was accomplished using two parametric models: (a) a modelindependent line-shape, hereafter named model 1 (M1) and (b) a three dimensional anisotropic Gaussian line-shape derived from a quasi-harmonic model, hereafter named model 2 (M2). The latter was most recently employed for the understanding of the local environment of hydrogen in polycrystalline ice ${ }^{47}$ and heavy water. ${ }^{48}$

In M1, the momentum distribution is given by the GaussLaguerre expansion ${ }^{34,43,49}$

$$
n_{\mathrm{M} 1}(p)=\frac{\exp \left(-\frac{p^{2}}{2 \sigma^{2}}\right)}{(\sqrt{2 \pi} \sigma)^{3}} \sum_{n} c_{n}(-1)^{n} L_{n}^{\frac{1}{2}}\left(\frac{p^{2}}{2 \sigma^{2}}\right),
$$

where $L_{n}^{\frac{1}{2}}$ are the generalized Laguerre polynomials, and $c_{n}$ the expansion coefficients from which, together with the standard deviation, $\sigma$, one can derive the momentum distribution lineshape.

In M2, the momentum distribution has been modelled using the spherical average of multivariate Gaussian distribution of the form ${ }^{47}$

$$
4 \pi p^{2} n_{\mathrm{M} 2}(p)=\left\langle\frac{\delta(p-|\mathbf{p}|)}{\sqrt{8 \pi^{3}} \sigma_{x} \sigma_{y} \sigma_{z}} \exp \left(-\frac{p_{x}{ }^{2}}{2 \sigma_{x}^{2}}-\frac{p_{y}{ }^{2}}{2 \sigma_{y}{ }^{2}}-\frac{p_{z}{ }^{2}}{2 \sigma_{z}^{2}}\right)\right\rangle,
$$

where $\sigma_{z}$ is along the direction of the $\mathrm{H}$ bond, and $\sigma_{x}$ and $\sigma_{y}$ are in the plane perpendicular to the direction of the $\mathrm{H}$ bond, i.e., the direction of the maximum value for the zero point energy. The parameter set $\sigma_{x, y, z}$, determines the anisotropy in the momentum distribution line-shape. Fig. 2 reports a sketch showing the orientation of the water molecules adsorbed on the GO surface and the axis reference system used for the anisotropic model M2.

It has to be stressed that although the M1 model represents the most general momentum distribution line-shape, it does not allow the separation of the effects of anharmonicity from those of anisotropy. ${ }^{32,50}$ Values of the standard deviations $\sigma$ and $\sigma_{\alpha}$, with $\alpha=x, y, z$, from the M1 and M2 models respectively, have been obtained by performing a global fit of the whole set of forward-angle spectra (see SM). Moreover, the hydrogen total mean kinetic energy, $\left\langle E_{\mathrm{K}}\right\rangle=3 \frac{\hbar^{2} \sigma^{2}}{2 m}$, and the directional components along the three spatial axes, $\left\langle E_{\mathrm{K}}\right\rangle_{\alpha}=\frac{\hbar^{2} \sigma_{\alpha}{ }^{2}}{2 m}$, have been evaluated and reported in Table 1 . From this table we can appreciate that both M1 and M2 models provide the same results for $\left\langle E_{\mathrm{K}}\right\rangle$ with little differences in directional $\left\langle E_{\mathrm{K}}\right\rangle_{\alpha}$ values between water and ice. Overall, the $\mathrm{H}$ dynamics of water and ice confined in 2D GO exhibits similar three dimensional anisotropy to that found in bulk water ${ }^{51}$ and ice. ${ }^{47}$ The latter is fingerprinted by the directional components of kinetic energy, $\left\langle E_{\mathrm{K}}\right\rangle_{\alpha}$, with values which are, however, different with respect to
Table 1 Values of the global-fit parameters on experimental measurements for water in GO at $T=293 \mathrm{~K}$ and at $20 \mathrm{~K}$

\begin{tabular}{lcc}
\hline & $293 \mathrm{~K}$ & \multicolumn{1}{c}{$20 \mathrm{~K}$} \\
\hline $\mathrm{M} 1$ & & \\
$\sigma\left[\AA^{-1}\right]$ & $5.01 \pm 0.03$ & $4.99 \pm 0.03$ \\
$c_{4}\left[E_{\mathrm{K}}\right\rangle[\mathrm{meV}]$ & $0.14 \pm 0.01$ & $0.12 \pm 0.1$ \\
& $156.1 \pm 2.0$ & $154.9 \pm 2.0$ \\
$\mathrm{M} 2$ & & \\
$\sigma_{x}\left[\AA^{-1}\right]$ & $3.4 \pm 0.5$ & $3.5 \pm 0.5$ \\
$\sigma_{y}\left[\AA^{-1}\right]$ & $4.6 \pm 0.5$ & $4.6 \pm 0.5$ \\
$\sigma_{z}\left[\AA^{-1}\right]$ & $6.4 \pm 0.2$ & $6.3 \pm 0.2$ \\
$\left\langle E_{\mathrm{K}}\right\rangle[\mathrm{meV}]$ & $156.1 \pm 2.0$ & $154.9 \pm 2.0$ \\
$\left\langle E_{\mathrm{K}}\right\rangle_{x}[\mathrm{meV}]$ & $24 \pm 6$ & $26 \pm 7$ \\
$\left\langle E_{\mathrm{K}}\right\rangle_{y}[\mathrm{meV}]$ & $45 \pm 9$ & $45 \pm 9$ \\
$\left\langle E_{\mathrm{K}}\right\rangle_{z}[\mathrm{meV}]$ & $86 \pm 12$ & $84 \pm 12$ \\
\hline
\end{tabular}

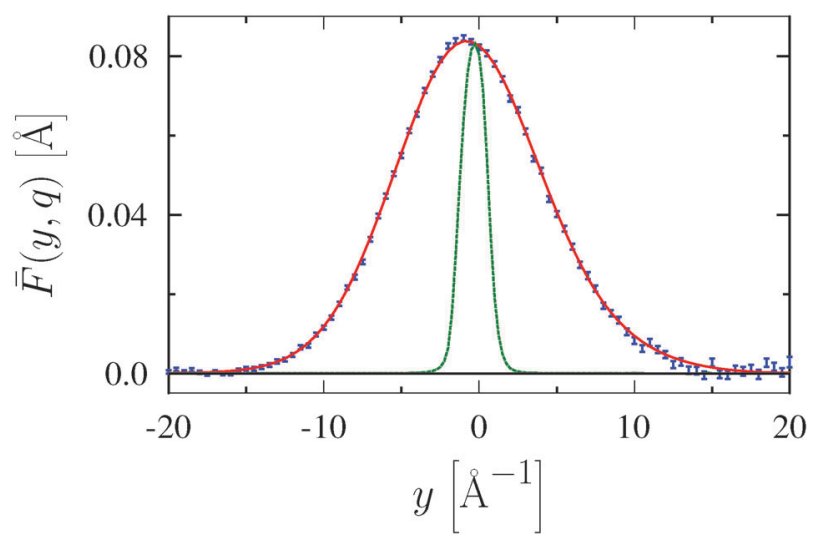

Fig. 1 Angle averaged hydrogen NCP, $\bar{F}(y, q)$, for water in GO sponges at $T=293 \mathrm{~K}$ (blue square with error bars). The fit using $M 2$ is plotted as a red line. The experimental resolution $R(y, q)$ is plotted as a green line.

those found in the bulk. The angle-averaged experimental lineshape for DINS data at $T=293 \mathrm{~K}, \bar{F}(y, q)$, is plotted in Fig. 1 together with fit line-shapes resulting from model M2.

In Table 2 a comparison is made between values for the $\left\langle E_{\mathrm{K}}\right\rangle$ of the present experiment and other DINS experiments on bulk and confined water and ice. These results are consistent with a picture where, for bulk water and ice, the kinetic energy is dominated by the ground state contributions, with the thermal contributions affecting mostly the high-temperature liquid upon approaching the supercritical phase. Differences in the ground state contributions between the solid and the liquid are evident, i.e., the $\left\langle E_{\mathrm{K}}\right\rangle$ value of the solid at $T=271 \mathrm{~K}$ is about $13 \mathrm{meV}$ higher than the value of the liquid at $T=300 \mathrm{~K}$ (see Table 2). Present analysis provides $\left\langle E_{\mathrm{K}}\right\rangle$ values for water at $T=293 \mathrm{~K}$ and ice at $T=20 \mathrm{~K} 2 \mathrm{D}$ confined in GO in excess of $13 \mathrm{meV}$ and $3.7 \mathrm{meV}$ compared to the corresponding values found in the bulk, respectively. Both values fingerprint the dynamics of water and ice molecules interacting with functional groups on the layers of the GO sponge.

The plot of radial momentum distributions, $4 \pi p^{2} n(p)$, corresponding to M2 for water and ice in GO is shown in Fig. 3. The high momentum components in $n(p)$ are highly sensitive to, and dominated by, the curvature of the effective 


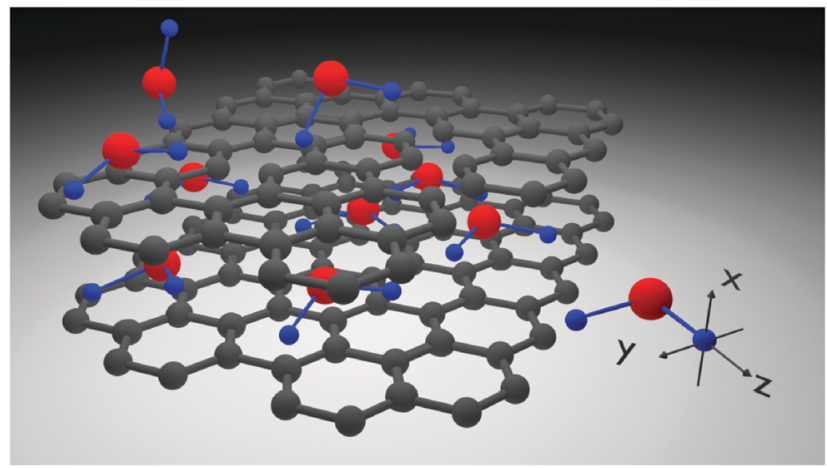

Fig. 2 Orientation of the water molecules adsorbed on the GO surface and the axis reference system used for the anisotropic model M2.

Table 2 The values of the mean kinetic energy resulting from the present experiment (italic), compared with previous measurements

\begin{tabular}{|c|c|c|}
\hline Sample & $T[\mathrm{~K}]$ & $\left\langle E_{\mathrm{K}}\right\rangle[\mathrm{meV}]$ \\
\hline Water $^{a}$ & 423 & $155.0 \pm 3$ \\
\hline Water in $G O$ & 293 & $156.1 \pm 2.0$ \\
\hline Water in xerogel pores $(24 \AA)^{b}$ & 293 & $187 \pm 3$ \\
\hline Water in xerogel pores $(82 \AA)^{b}$ & 293 & $156 \pm 3$ \\
\hline Ice bulk ${ }^{c}$ & 271 & $156 \pm 2$ \\
\hline Water bulk ${ }^{a}$ & 300 & $143 \pm 3$ \\
\hline Ice bulk $^{d}$ & 71 & $152.8 \pm 2$ \\
\hline Ice in $G O$ & 20 & $154.9 \pm 2.0$ \\
\hline Ice bulk ${ }^{d}$ & 5 & $150.9 \pm 1.5$ \\
\hline
\end{tabular}

proton potential,,$^{53-56}$ i.e., closely related to the hydrogen bond strength. The $n(p)$ in a frame attached to an individual water molecule is well described by a harmonic anisotropic Gaussian line-shape, with the transverse momentum width slightly more than one half the width along the stretch mode direction. The tail of the $n(p)$ distributions is due to the momentum along the bond direction, since the proton is most tightly bound in this direction, tight binding implying high momentum width. From Fig. 3 one can appreciate that the momentum width of the $n(p)$ line-shape of water adsorbed in GO at $T=293 \mathrm{~K}$ is clearly

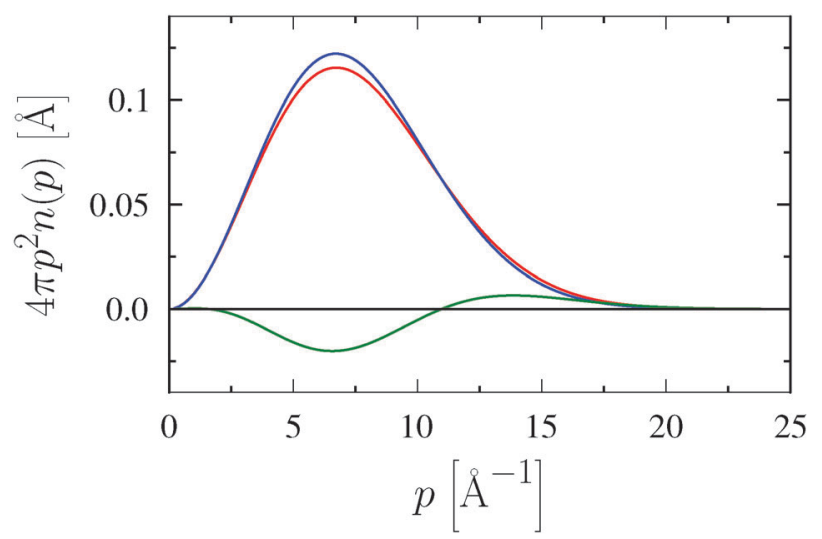

Fig. $3 n(p)$ for water in GO sponges at $T=293 \mathrm{~K}$ (red line) and bulk water at $T=300 \mathrm{~K}$ (blue line); the difference of the two line-shapes, magnified by three times, is plotted as the green line. expanded from about $11 \AA^{-1}$ as compared to bulk water at $T=300 \mathrm{~K}$. This finding, similar to what was observed in water at $T=423 \mathrm{~K}^{53}$ (see Table 2), implies that hydrogen bonds are expected to be significantly weaker than in bulk water. The momentum width of the $n(p)$ line-shape for ice in GO at $T=20 \mathrm{~K}$ extends a little less, mirroring an $\mathrm{OH}$ bond network stronger than in water at $T=293 \mathrm{~K}$, although weaker than the value expected for bulk ice at the same temperature.

\section{Conclusions}

The overall $\mathrm{H}$ dynamics of water and ice confined in 2D GO exhibits an anisotropy of the momentum distribution $n(p)$, very similar to what is found in bulk water. In GO, water molecules are restrained in layered geometries, with interlayer distances down to $12 \AA{ }^{57}$ This length scale is comparable to the scales of the tetrahedral structural motifs in bulk water. The water response to the perturbation due to this nanometric confinement results in an increase of the hydrogen mean kinetic energy with respect to the bulk. The increase is approximately $13 \mathrm{meV}$ at room temperature and approximately $4 \mathrm{meV}$ at $20 \mathrm{~K}$. Moreover, the hydrogen kinetic energy of water in GO shows a negligible temperature dependence in contrast to the bulk. It appears that confinement in $\mathrm{GO}$ on one hand induces a higher proton localisation, with increased $\left\langle E_{\mathrm{K}}\right\rangle$, and on the other hand preserves the hydrogen dynamics from temperature effects. Likewise, $n(p)$ functions show harmonic anisotropic lineshapes. These findings are ascribed to changes of the proton dynamics induced by the interaction between interfacial water and ice and the confining hydrophilic surface. Due to the negligible DINS signal from the structural hydrogens of the dry GO sponges, the DINS results indicate that changes on $\left\langle E_{\mathrm{K}}\right\rangle$ are mainly due to steric confinement and that the interactions of water with the functional groups on GO play a negligible role. This result is an important piece of information in view of a thorough understanding of the hydrogen bonding dynamics in GO.

Whatever the origin of the interaction, $\mathrm{H}$ dynamics in water confined in 2D GO is quantitatively different from that of water confined in other nanoporous hydrophilic surfaces, such as xerogel, MCM41 and nanotubes, and unlike any other form of water observed so far. The present results support a picture where the $\mathrm{OH}$ bonds of water and ice in GO are softer than in bulk water with the energy of the $\mathrm{OH}$ expected to be red shifted with a corresponding broadening of the spectral peak.

\section{Acknowledgements}

This work was supported within the CNR-STFC Agreement No. 06/20018 concerning collaboration in scientific research at the spallation neutron source ISIS. K. P. Loh thanks funding support from NRF Investigatorship Award "Graphene oxide a new class of catalytic, ionic and molecular sieving materials NRF12015-01". 


\section{References}

1 M. Bellissent-Funel, Hydration Processes in Biology: Theoretical and Experimental Approaches, IOS Press, 1999.

2 O. Mishima and H. E. Stanley, Nature, 1998, 396, 329-335.

3 C.-Y. Lee, J. A. McCammon and P. J. Rossky, J. Chem. Phys., 1984, 80, 4448-4455.

4 P. A. Thiel and T. E. Madey, Surf. Sci. Rep., 1987, 7, 211-385. 5 P. G. Debenedetti, J. Phys.: Condens. Matter, 2003, 15, 1669. 6 V. Garbuio, C. Andreani, S. Imberti, A. Pietropaolo, G. F. Reiter, R. Senesi and M. A. Ricci, J. Chem. Phys., 2007, 127, 4501.

7 F. Mallamace, M. Broccio, C. Corsaro, A. Faraone, L. Liu, C.-Y. Mou and S.-H. Chen, J. Phys.: Condens. Matter, 2006, 18, 2285.

8 C. Pantalei, R. Senesi, C. Andreani, P. Sozzani, A. Comotti, S. Bracco, M. Beretta, P. E. Sokol and G. Reiter, Phys. Chem. Chem. Phys., 2011, 13, 6022-6028.

9 W. Doster, A. Bachleitner, R. Dunau, M. Hiebl and E. Luscher, Biophys. J., 1986, 50, 213-219.

10 G. Sartor, A. Hallbrucker, K. Hofer and E. Mayer, J. Phys. Chem., 1992, 96, 5133-5138.

11 K. Morishige and K. Nobuoka, J. Chem. Phys., 1997, 107, 6965-6969.

12 S.-H. Chen, P. Gallo and M.-C. Bellissent-Funel, Can. J. Phys., 1995, 73, 703.

13 J.-M. Zanotti, M.-C. Bellissent-Funel and S.-H. Chen, Phys. Rev. E: Stat. Phys., Plasmas, Fluids, Relat. Interdiscip. Top., 1999, 59, 3084-3093.

14 L. D. Gelb, K. E. Gubbins, R. Radhakrishnan and M. SliwinskaBartkowiak, Rep. Prog. Phys., 2000, 63, 727.

15 M. C. Bellissent-Funel, S. Longeville, J. M. Zanotti and S. H. Chen, Phys. Rev. Lett., 2000, 85, 3644.

16 F. Venturini, P. Gallo, M. A. Ricci, A. R. Bizzarri and S. Cannistraro, J. Chem. Phys., 2001, 114, 10010-10014.

17 R. Zangi, J. Phys.: Condens. Matter, 2004, 16, 5371.

18 S.-H. Chen, L. Liu, E. Fratini, P. Baglioni, A. Faraone and E. Mamontov, Proc. Natl. Acad. Sci. U. S. A., 2006, 103, 9012-9016.

19 P. G. Debenedetti, Metastable Liquids: Concepts and Principles, Princeton University Press, 1997.

20 D. Chandler, Nature, 2005, 437, 640-647.

21 J. Marti, G. Nagy, M. C. Gordillo and E. Guàrdia, J. Chem. Phys., 2006, 124, 094703.

22 G. Nagy, M. C. Gordillo, E. Guàrdia and J. Marti, J. Phys. Chem. B, 2007, 111, 12524-12530.

23 B. C. Brodie, Ann. Chim. Phys., 1860, 466, 59.

24 R. Yazami and P. Touzain, Synth. Met., 1985, 12, 499-503.

25 T. Cassagneau and J. H. Fendler, Adv. Mater., 1998, 10, 877-881.

26 T. Hwa, E. Kokufuta and T. Tanaka, Phys. Rev.A: At., Mol., Opt. Phys., 1991, 44, 2235.

27 X. Wen, C. W. Garland, T. Hwa, M. Kardar, E. Kokufuta, Y. Li, M. Orkisz and T. Tanaka, Nature, 1992, 355, 426-428.

28 F. F. Abraham and M. Goulian, EPL, 1992, 19, 293.

29 J. Lu, A. H. C. Neto and K. P. Loh, Nat. Commun., 2012, 3, 823.

30 Y. Zheng, C. Su, J. Lu and K. P. Loh, Angew. Chem., Int. Ed., 2013, 52, 8708-8712.
31 H. Bai, C. Li, X. Wang and G. Shi, Chem. Commun., 2010, 46, 2376-2378.

32 C. Andreani, D. Colognesi, J. Mayers, G. F. Reiter and R. Senesi, Adv. Phys., 2005, 54, 377-469.

33 G. F. Reiter, R. Senesi and J. Mayers, Phys. Rev. Lett., 2010, 105, 148101.

34 G. F. Reiter, J. Mayers and J. Noreland, Phys. Rev. B: Condens. Matter Mater. Phys., 2002, 65, 104305.

35 P. C. Hohenberg and P. M. Platzman, Phys. Rev., 1966, 152, 198-200.

36 V. I. Gol'danskii, Sov. Phys.-JETP, 1957, 4, 604.

37 G. K. Ivanov and Y. S. Sayasov, Sov. Phys.-Dokl., 1964, 9, 171.

38 A. Parmentier, J. J. Shephard, G. Romanelli, R. Senesi, C. G. Salzmann and C. Andreani, J. Phys. Chem. Lett., 2015, 6, 2038-2042.

39 M. Krzystyniak, M. A. Adams, A. Lovell, N. T. Skipper, S. M. Bennington, J. Mayers and F. Fernandez-Alonso, Faraday Discuss., 2011, 151, 171-197.

40 R. Senesi, C. Andreani, Z. Bowden, D. Colognesi, E. Degiorgi, A. L. Fielding, J. Mayers, M. Nardone, J. Norris, M. Praitano, N. J. Rhodes, W. G. Stirling, J. Tomkinson and C. Uden, Phys. $B, 2000,276,200-201$.

41 J. M. F. Gunn, C. Andreani and J. Mayers, J. Phys. C: Solid State Phys., 1986, 19, L835-L840.

42 G. B. West, Phys. Rep., 1975, 18, 263-323.

43 G. Reiter and R. Silver, Phys. Rev. Lett., 1985, 54, 1047-1050. 44 G. I. Watson, J. Phys.: Condens. Matter, 1996, 8, 5955-5975. 45 A. Pietropaolo and R. Senesi, Phys. Rep., 2011, 508, 45-90.

46 J. Mayers and G. Reiter, Meas. Sci. Technol., 2012, 23, 045902.

47 D. Flammini, A. Pietropaolo, R. Senesi, C. Andreani, F. McBride, A. Hodgson, M. A. Adams, L. Lin and R. Car, J. Chem. Phys., 2012, 136, 024504.

48 G. Romanelli, M. Ceriotti, D. E. Manolopoulos, C. Pantalei, R. Senesi and C. Andreani, J. Phys. Chem. Lett., 2013, 4, 3251-3256.

49 A. Pietropaolo, R. Senesi, C. Andreani, A. Botti, M. A. Ricci and F. Bruni, Phys. Rev. Lett., 2008, 100, 127802.

50 G. F. Reiter, J. C. Li, J. Mayers, T. Abdul-Redah and P. Platzman, Braz. J. Phys., 2004, 34, 142-147.

51 C. Andreani, G. Romanelli and R. Senesi, Chem. Phys., 2013, 427, 106-110.

52 R. Senesi, G. Romanelli, M. Adams and C. Andreani, Chem. Phys., 2013, 427, 111-116.

53 C. Pantalei, A. Pietropaolo, R. Senesi, S. Imberti, C. Andreani, J. Mayers, C. Burnham and G. Reiter, Phys. Rev. Lett., 2008, 100, 177801.

54 C. J. Burnham, G. F. Reiter, J. Mayers, T. Abdul-Redah, H. Reichert and H. Dosch, Phys. Chem. Chem. Phys., 2006, 8, 3966.

55 J. A. Morrone, V. Srinivasan, D. Sebastiani and R. Car, J. Chem. Phys., 2007, 126, 234504.

56 C. J. Burnham, D. J. Anick, P. K. Mankoo and G. F. Reiter, J. Chem. Phys., 2008, 128, 154519.

57 A. Buchsteiner, A. Lerf and J. Pieper, J. Phys. Chem. B, 2006, 110, 22328. 
Probing the 2D confinement on hydrogen dynamics in water and ice adsorbed in graphene oxide sponges

\section{Electronic Supplementary Information}

Giovanni Romanelli, ${ }^{* a}$ Roberto Senesi, ${ }^{a b}$ Xuan Zhang, ${ }^{c}$ Kian Ping Loh, ${ }^{c}$ Carla Andreani ${ }^{a}$

\section{Experimental Methods}

\subsection{Sample Preparation}

The graphene oxide (GO) is prepared by hydrothermal oxidation in a sealed autoclave. Typically, $50 \mathrm{mg}$ graphene are cooled in a $50 \mathrm{ml}$ autoclave at $4 \mathrm{C}$ for 1 hour before experiment. The pre-cooled $\mathrm{KMnO}_{4}(1 \mathrm{~g})$ and concentrated sulfuric acid $(20 \mathrm{ml})$ are slowly added into the autoclave under ice bath. The autoclave is tightly sealed and kept in the fridge for 2 hours before heating in oven at $150 \mathrm{C}$ for 2 hours. The resulted GO are thoroughly washed with deionized water and dried in oven overnight (see Figure 2). To synthesize the graphene hydrogel, purified GO is dispersed in $20 \mathrm{ml}$ water $(2 \mathrm{mg} / \mathrm{ml})$ and sealed in an autoclave (50 ml volume). The autoclave is heated in oven at 160 $\mathrm{C}$ for 12 hours. The cylinder-shaped hydrogel is thoroughly washed with water and freeze-dried for 2 days.

\section{$1.2 \quad$ FTIR characterisation of dry GO}

The FTIR spectrum of GO sponge, which was used for the DINS measurements, reported in Figure 1 , shows the presence of hydroxyl, epoxide and carbonyl groups. No obvious peak for $\mathrm{C}-\mathrm{H}$ band is observed. Therefore, the hydrogen amount in the GO sponge is very small.

\subsection{Hydration Procedure}

The freeze-dried cylindrical GO gel was put in a glass bottle and soaked in deionized water for 12 hour prior to the DINS experiment. The glass bottle was fully filled with water and properly capped to avoid water evaporation.

The GO sponge has a strong ability to absorb water. In the present experiment, the weight of dry GO was $0.168 \mathrm{~g}$. The specific surface area of GO sponge varies 180 to $800 \mathrm{~m} 2 / \mathrm{g}$, depending on different synthetic methods and the GO exfoliation degree. The GO sponge used in the DINS experiment was prepared with a method described in Ref. ${ }^{1}$ with a reported surface area of $414 \mathrm{~m}^{2} / \mathrm{g}$. The sample with water fully absorbed in the GO sponge had a total weight of $1.340 \mathrm{~g}$. Weights

* Current address: ISIS Facility, Rutherford Appleton Laboratory, Chilton, Didcot, Oxfordshire OX11 0QX, United Kingdom; E-mail: giovanni.romanelli@stfc.ac.uk

a Università degli Studi di Roma "Tor Vergata", Dipartimento di Fisica and Centro NAST, Via della Ricerca Scientifica 1, 00133 Roma, Italy

${ }^{b}$ Consiglio Nazionale delle Ricerche, CNR-IPCF, Sezione di Messina, Italy

${ }^{c}$ Graphene Research Centre and Centre for Advanced 2D Materials, Department of Chemistry, National University of Singapore, 3 Science Drive 3, Singapore 117543 


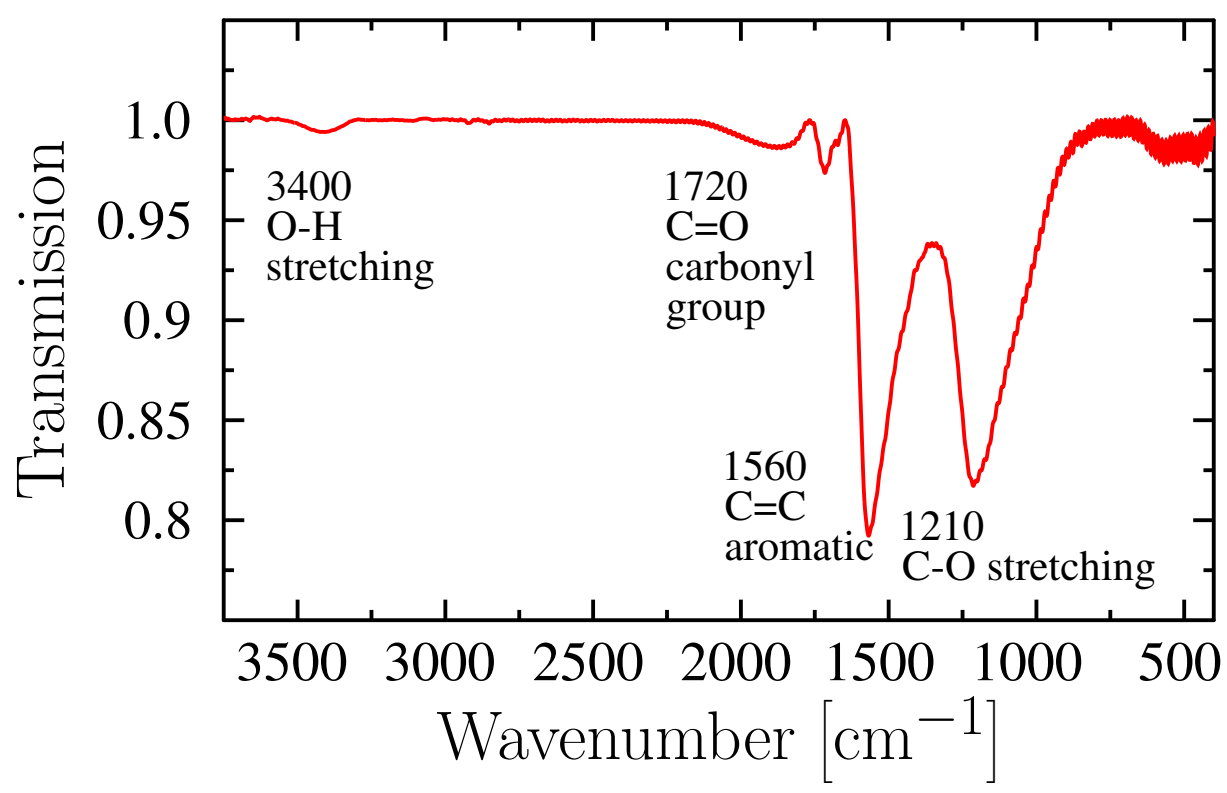

Fig. 1 FTIR spectrum of the dry GO sponge.

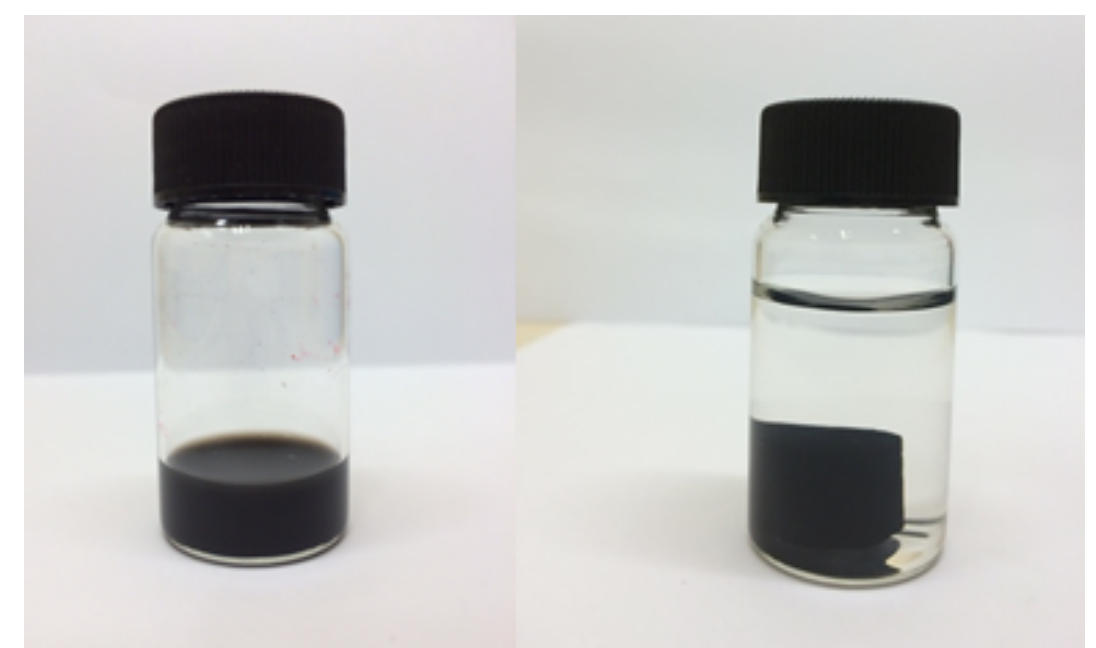

Fig. 2 GO solution (left) and GO hydrogel soaked in water (right). 


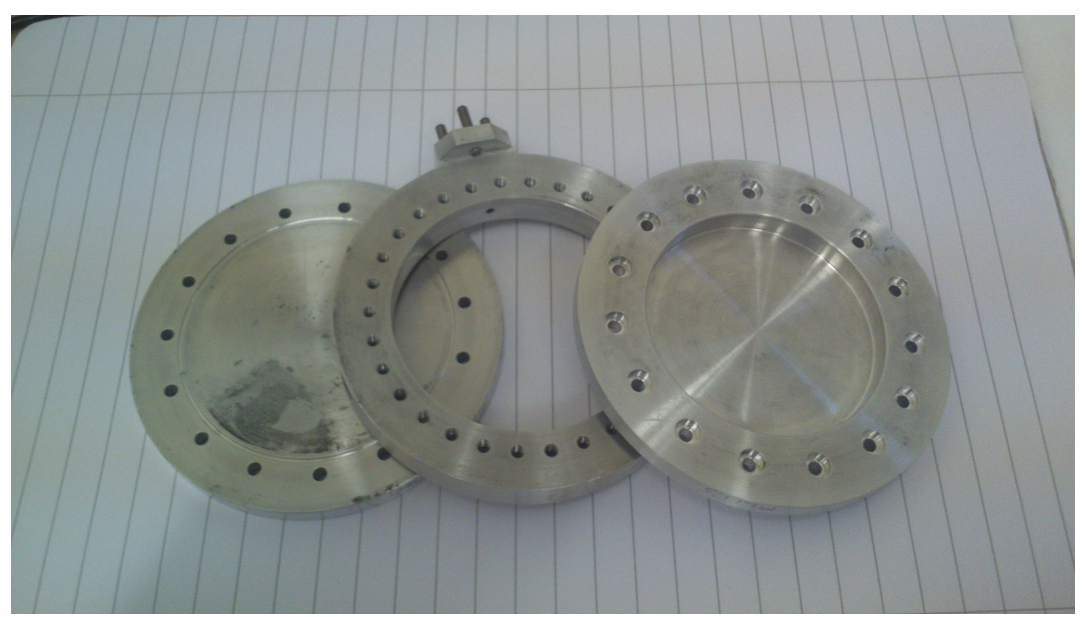

Fig. 3 Aluminium sample container used for ther DINS measurements.

of the hydrated sample and the dry GO were $1.172 \mathrm{~g}$ and $0.168 \mathrm{~g}$, respectively, corresponding to approximately $87 \mathrm{wt} \%$ of adsorbed water.

\subsection{Sample transfer into the sample containers}

The hydrated GO sponge sample consisted of three GO sponges (total masses $0.168 \mathrm{~g}$ of dry GO plus $1.172 \mathrm{~g}$ of water), which were located inside a square $\mathrm{Al}$ sachet (and placed in the centre of the $\mathrm{Al}$ sample container (see figure 3 below). The inner volume of the sample container was $1 \mathrm{~cm}$ width and $7 \mathrm{~cm}$ diameter. To maximise neutron signal from the sample the diagonal of the squared sachet was $7 \mathrm{~cm}$, equal to diameter of sample container.

\subsection{DINS measurements}

VESUVIO is an inverse geometry spectrometer, i.e., for each scattering angle, $\theta$, the final energy of the scattered neutrons, $E_{1}$, is selected using a $A u$ resonance filter which absorbs neutrons in a narrow range of energies ${ }^{2}$ with the scattering signal recorded by individual detectors. VESUVIO operates for DINS experiments in the IA regime where the incident neutron wavelengths are much less than the inter-atomic spacing and thus atoms scatter incoherently, with scattered intensity being the sum of intensities from individual atoms in the sample. The count rate as a function of the time of flight (t.o.f.) $t$ yields:

$$
C(t)=\sqrt{\frac{8 E_{0}^{3}}{m_{N} L_{0}^{2}}} I\left(E_{0}\right) D\left(E_{1}\right)\left(\sum_{M} N_{M} \frac{d^{2} \sigma_{M}}{d \Omega d E_{1}}\right) d \Omega
$$

where $I\left(E_{0}\right) d E_{0}$ is the number of incident neutrons $\mathrm{s}^{-1}$ with energies between $E_{0}$ and $E_{0}+d E_{0}$, $D\left(E_{1}\right)$ is the probability that a neutron of energy $E_{1}$ is detected, $m_{N}$ is the neutron mass, $L_{0}$ is the moderator - sample distance, $N_{M}$ is the number of atoms of mass $M$ in the sample and $\frac{d^{2} \sigma_{M}}{d \Omega d E_{1}}$ is the partial differential cross-section for the struck nucleide. In the forward direction scattered neutrons are detected by Yttrium Aluminum Perovskite (YAP) scintillators ${ }^{3}$, located at a distance $L_{1}$, ranging between $0.5 \mathrm{~m}$ and $0.75 \mathrm{~m}$ from sample position, in the angular range $32.75^{\circ}$ to $72.5^{\circ}$. In the backward direction scattered neutrons are detected by $\mathrm{Li}^{6}$ scintillators, located at a distance 


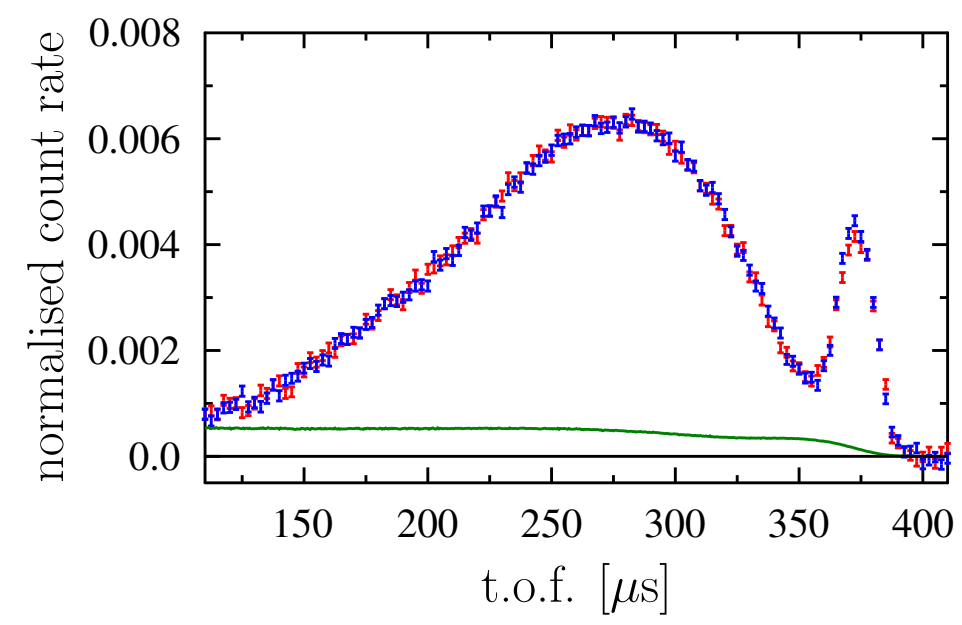

Fig. 4 Angle averaged of the raw t.o.f. spectra for water and ice adsorbed in GO sponges at $T=293 \mathrm{~K}$ (red line) and $T=$ $20 \mathrm{~K}$ (blue line) and MS component (green line).

ranging between $0.46 \mathrm{~m}$ and $0.67 \mathrm{~m}$ from sample position, in the angular range $130^{\circ}$ to $163^{\circ}$. At each scattering angle the energy of the scattered neutrons, $E_{1}$, is selected by using $\mathrm{Au}$ analyzer foils $\left(E_{1}=4897 \mathrm{meV}\right)$. The instrument operates using the Foil Cycling (FC) technique, in forward scattering $^{2,4}$, and the Double Difference (DD) technique ${ }^{5}$, in backward scattering in the range of wave vector and energy transfers is $27 \AA^{-1} \leq q \leq 230 \AA^{-1}$ and $2.5 \mathrm{eV} \leq \hbar \omega \leq 65 \mathrm{eV}$, respectively and $10^{-3} \mathrm{eV} \leq \hbar \omega \leq 10^{4} \mathrm{eV}$, respectively ${ }^{6,7}$.

Multilayer stacked graphene oxide layers, in the range from 6 to $12 \AA$, ensured ice formation in these layer by layer stacked layers as well as water trapping ability in 2D and the NCS signal strengths. The sample synthesis has been carried out to guarantee a number of more than $10^{21}$ water molecules trapped in the matrices, allowing a reliable counting statistics at both temperatures.

The integrated proton current for DINS data yielded $I=4220 \mu \mathrm{Ah}$ for $\mathrm{T}=293 \mathrm{~K}, I=3960 \mu \mathrm{Ah}$ for $\mathrm{T}=20 \mathrm{~K}$.

Raw t.o.f. data of individual detectors for water and ice in GO have been corrected by $\gamma$ background and multiple scattering (MS) and Al sample container contributions using a Monte Carlo simulation and the simulated signals subtracted from the experimental data. Angle averaged sum over all forward scattering detectors of t.o.f. data for water and ice adsorbed in GO are reported in Figure 4 together with MS contribution. From this figure one can see the hydrogen signal peaked between $250-300 \mu$ s and the $\mathrm{Al}$ signal peaked between $350-400 \mu$ s. An example of a t.o.f. corrected spectrum for water in GO sponges at $T=293 \mathrm{~K}$, for an individual detector, $\theta=35$ degrees, is reported in Figure 5. Averaged raw experimental hydrogen NCP and corrected NCP are reported in Figure 6 and Figure 7, respectively. Figure 8 reports the raw data for hydrated and dry GO in $y$ space, respectively; this latter figure shows that the contribution of hydrogen signal from the dry $\mathrm{GO}$ is negligible in comparison with the signal from the hydrated sample.

The partial differential cross-section from Eq. (1) can be expressed as a function of the NCP, $J(y)$ as follows:

$$
\frac{d^{2} \sigma_{M}}{d \Omega d E_{1}}=b^{2}\left(\frac{E_{1}}{E_{0}}\right)^{1 / 2} \frac{M}{\hbar q} J(y) .
$$




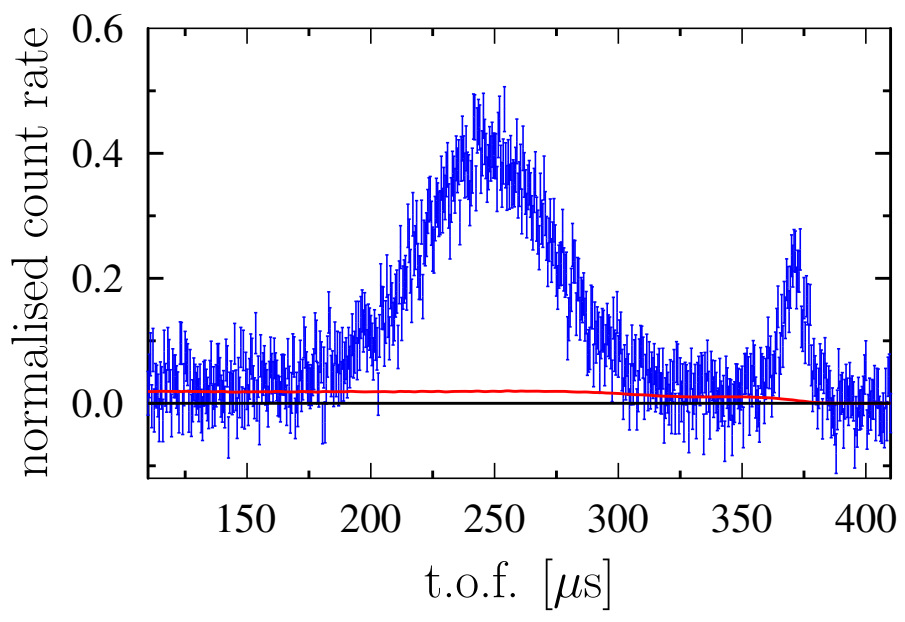

Fig. 5 Individual DINS spectrum in t.o.f. for water in GO sponges at $T=293 \mathrm{~K}$ (blue line), $\theta=35$ degrees, corrected for $\gamma$-background and multiple scattering (MS) and Al sample container contributions. The MS contribution is plotted in red line.

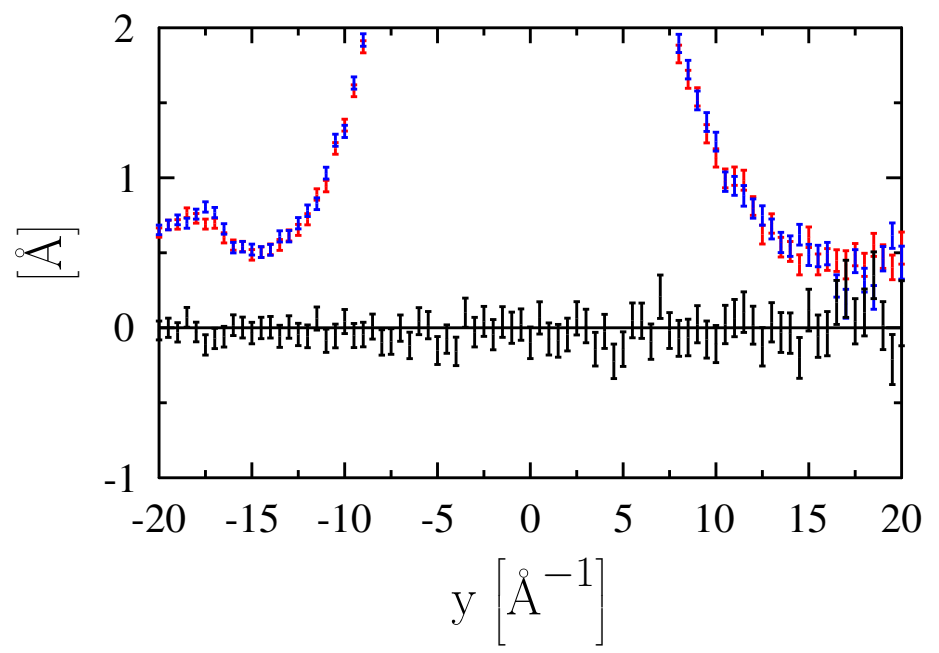

Fig. 6 Angle averaged raw hydrogen NCP for water and ice in GO sponges at $T=293 \mathrm{~K}$ (red square with error bars) and $T$ $=20 \mathrm{~K}$ (blue square with error bars) and the difference signal. 


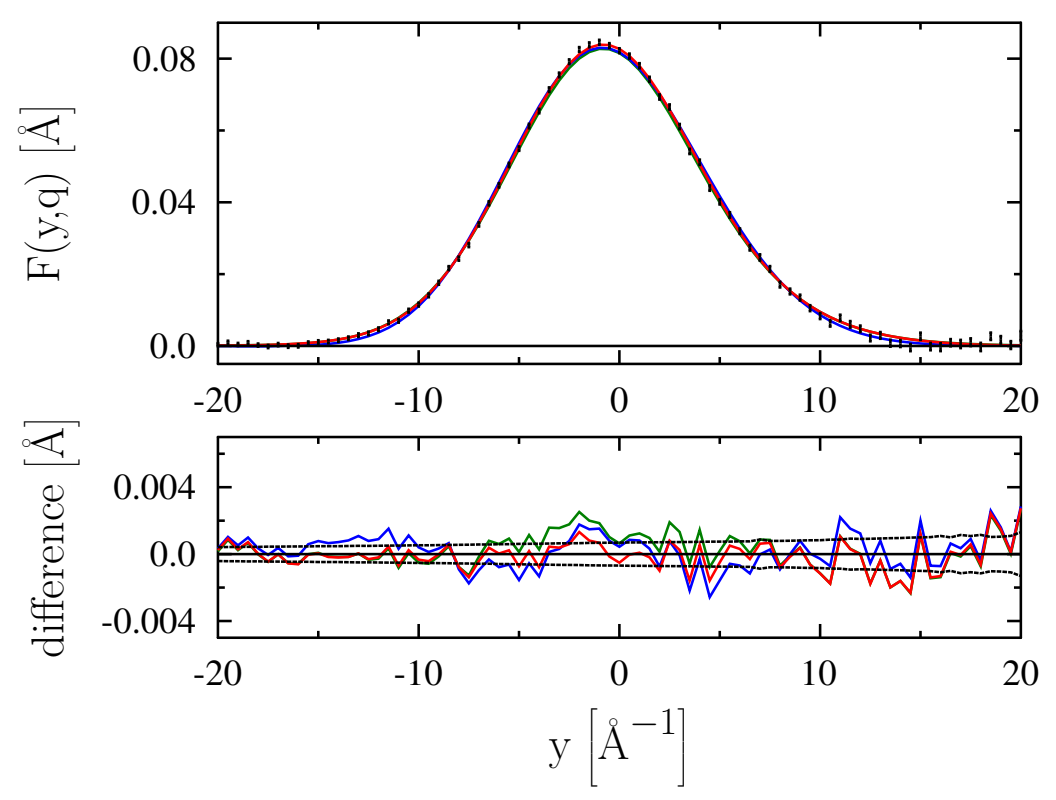

Fig. 7 Averaged experimental response function, $F(y, q)$, (black dots with error bars) at $T=293 \mathrm{~K}$ and line shapes resulting from fit using model M1, M2 and simple Gaussian lineshape. Upper panel: multivariate-Gaussian model (M2) is reported as a (green line). Lower panel: difference between lineshape resulting from model M2 and $F(y, q)$. Similar comparison is reported for Gauss-Hermite model (M1) up to the first polynomial (red line) and the with no polynomials (blue line).

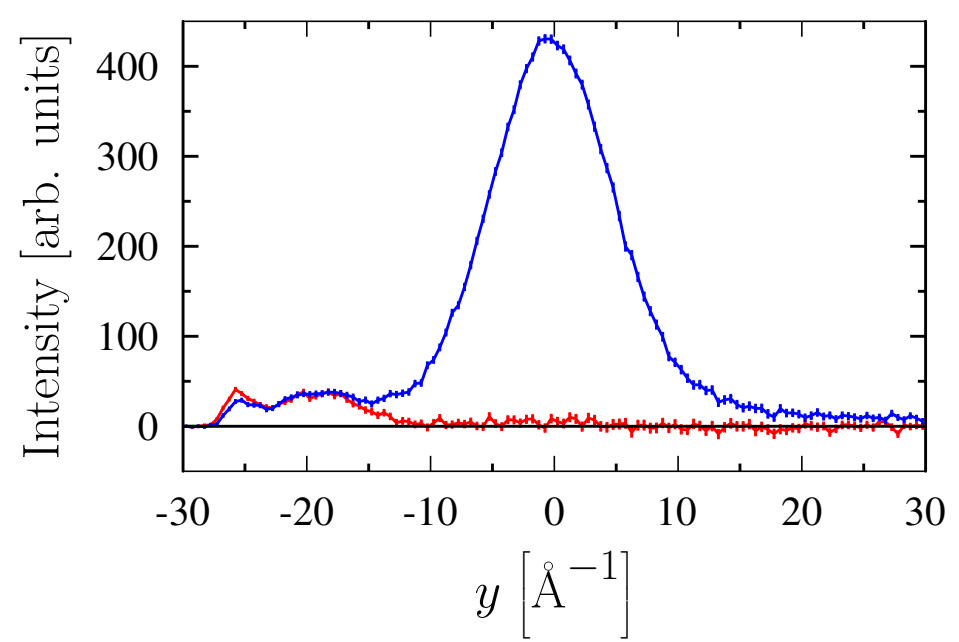

Fig. 8 Raw data for hydrated (blue line and dots with error bars) and dry (red line and dots with error bars) GO in $y$-space. 
Due to the finite $q$ values in the scattering process the NCP at each detector retains the $q$ dependence, expressed by the function $F(y, q)$. The latter includes a further broadening due to experimental resolution function, $R(y, q)$. The $F(y, q)$ function is related to the count rate via the expression:

$$
F(y, q)=\frac{B M}{E_{0} I\left(E_{0}\right)} q C(t)
$$

where $B$ is a constant taking into account: the detector solid angle, its efficiency at $E=E_{1}$, the time-energy Jacobian, the free-atom neutron cross section and the number of particles hit by the neutron beam. DINS data sets at all temperatures have been $y$-scaled according to Eq. (3).

In order to derive the $n(p)$, a line-shape analysis of $F(y, q)$ has been performed using models M1 and M2. The defining equations for the NCP related to a Gauss-Laguerre momentum distribution has the form of a Gauss-Hermite expansion

$$
J_{M 1}(y)=\frac{e^{\frac{-y^{2}}{2 \sigma^{2}}}}{\sqrt{2 \pi} \sigma}\left[1+\sum_{n=2}^{\infty} \frac{c_{2 n}}{2^{2 n} n !} H_{2 n}\left(\frac{y}{\sqrt{2} \sigma}\right)\right]
$$

and the NCP in the case of the multivariate Gaussian distribution has the form

$$
J_{M 2}(y)=\frac{1}{\sqrt{2 \pi} \sigma_{x} \sigma_{y} \sigma_{z}} \int_{\Omega} \frac{d \Omega}{4 \pi} \exp \left[-\frac{y^{2}}{2 S^{2}(\theta, \phi)}\right] S^{2}(\theta, \phi)
$$

with

$$
\frac{1}{S^{2}(\theta, \phi)}=\sin ^{2} \theta\left(\frac{\cos ^{2} \phi}{\sigma_{x}^{2}}+\frac{\sin ^{2} \phi}{\sigma_{y}^{2}}\right)+\frac{\cos ^{2} \theta}{\sigma_{z}^{2}}
$$

For both the models, the correction to the Impulse Approximation (IA) due to the Final State Effects (FSE) has been taken into account as an additive contribution of the form

$$
J(y, q)=J_{I A}(y)+\Delta J(y, q)=\left(1-A_{3}(q) \frac{\partial^{3}}{\partial y^{3}}\right) J_{I A}(y)
$$

where $A_{3}(q)=\frac{\sigma^{4}}{9 q}$.

The model fitting function has been obtained taking a numerical convolution of $J(y, q)$ with the experimental resolution $R(y, q)$, obtaining $F^{t h}(y, q)=J(y, q) \star R(y, q)$. Individual detectors have been fitted simultaneously via the $F^{t h}(y, q)$ line-shape in order to obtain the values of $\sigma$ and $c_{4}$ in the case of M1, and $\sigma_{x}, \sigma_{y}$ and $\sigma_{z}$ in the case of M2. The fitting parameters have been deduced by minimization the value:

$$
\chi^{2}=\sum_{l} \sum_{i} \frac{\left(F_{l}^{t h}\left(y_{i}, q_{i}\right)-F_{l}^{\exp }\left(y_{i}, q_{i}\right)\right)^{2}}{\epsilon_{l, i}^{2}}
$$

where $l$ labels the considered detector.

\section{References}

[1] Y. Xu, Z. Lin, X. Huang, Y. Liu, Y. Huang,X. Duan, ACS Nano, 74042 (2013). 
[2] R. Senesi, C. Andreani, Z. Bowden, D. Colognesi, E. Degiorgi, A. L. Fielding, J. Mayers, M. Nardone, J. Norris, M. Praitano, N. J. Rhodes, W. G. Stirling, J. Tomkinson and C. Uden, Physica B, 276 200-201 (2000)

[3] M. Tardocchi, A. Pietropaolo, C. Andreani, A. Bracco, A. DAngelo, G. Gorini, S. Imberti, R. Senesi, N. J. Rhodes, E. M. Schooneveld, Nucl. Instr. Meth. A 526, 477 (2004)

[4] E. M. Schooneveld, J. Mayers, N. J. Rhodes, A. Pietropaolo, C. Andreani, R. Senesi, G. Gorini, E. Perelli Cippo, M. Tardocchi, Rev. Sci. Instr. 77, 095103 (2006).

[5] C. Andreani, D. Colognesi, E. Degiorgi, A. Filabozzi, M. Nardone, E. Pace, A. Pietropaolo and R. Senesi, Nucl. Inst. Meth. A 497, 535-549 (2003)

[6] Reiter, G. F., Mayers J., and Noreland, J., Phys. Rev. B 65, 104305 (2002)

[7] Andreani, C., Colognesi, C., Mayers, J., Reiter, G. F. and Senesi, R. Adv., Phys. 54,377 (2005) 